\title{
BRIEF COMMUNICATION \\ Hydrogen bonding: a mechanism for tuning electronic and optical properties of hybrid organic-inorganic frameworks
}

\author{
Fedwa El-Mellouhi ${ }^{1}$, El Tayeb Bentria ${ }^{1}$, Asma Marzouk ${ }^{1}$, Sergey N Rashkeev ${ }^{1}$, Sabre Kais ${ }^{1,2,3}$ and Fahhad H Alharbi ${ }^{1,2}$
}

The field of hybrid inorganic-organic framework materials is one of the fastest growing fields in materials science because their enormous structural and chemical diversity presents great opportunities for creating many technologically relevant properties. One of the most important issues is controlling and tuning the structural, optical, thermal, mechanical and electronic properties of these complex materials by varying their chemistry, fabrication techniques and preparation conditions. Here we demonstrate that significant progress in this area may be achieved by introducing structural elements that form hydrogen bonds with the environment. Considering hybrid framework materials with different structural ordering containing protonated sulfonium cation $\mathrm{H}_{3} \mathrm{~S}^{+}$and electronegative halogen anions $\left(\mathrm{I}^{-}, \mathrm{Br}^{-}, \mathrm{Cl}^{-}\right.$and $\left.\mathrm{F}^{-}\right)$, we found that hydrogen bonding increases the structural stability of the material and may be used for tuning electronic states near the bandgap. We suggest that such a behaviour has a universal character and should be observed in hybrid inorganic-organic framework materials containing protonated cations. This effect may serve as a viable route for optoelectronic and photovoltaic applications.

npj Computational Materials (2016) 2, 16035; doi:10.1038/npjcompumats.2016.35; published online 4 November 2016

\section{INTRODUCTION}

In the past few years, hybrid organic-inorganic frameworks have aroused great interest due to the rich variety of their physical and chemical properties and potential application in nanotechnologies. These systems are prepared by binding multi-dentate organic molecules to multi-coordinate metal complexes and provide unlimited combinations of structures and properties, which enables to target design of materials for many applications. ${ }^{1}$ In some cases, the binding may also be achieved and/or assisted by other mechanisms such as halogen bonding ${ }^{2,3}$ or hydrogen bonding. ${ }^{4}$ On one side, porous hybrid framework materials for applications in chemical sensing, ${ }^{5}$ gas storage $^{6}$ and catalysis ${ }^{7}$ were developed. On the other side, there is a great interest to develop dense hybrid metal-organic systems for applications in optical and semiconductor devices and batteries. ${ }^{8}$ Some of dense hybrid frameworks such as $[\mathrm{AmH}] \mathrm{M}$ $(\mathrm{HCOO})_{3}$ adopt the $\mathrm{ABX}_{3}$ perovskite architecture $(\mathrm{A}=$ protonated amine, $[\mathrm{AmH}]^{+}, \mathrm{B}=\mathrm{M}^{2+}$ is a divalent metal, and $\mathrm{X}=\mathrm{HCOO}^{-}$is the formate anion) and exhibit a wide range of ferroelectric and multiferroic properties. ${ }^{9,10}$

Another class of hybrid perovskites of the composition [AmH] $\mathrm{MX}_{3}\left(\mathrm{M}=\mathrm{Sn}^{2+}, \mathrm{Pb}^{2+}\right.$ and $\left.\mathrm{X}=\mathrm{I}^{-}, \mathrm{Br}^{-}, \mathrm{Cl}^{-}\right)$, which were known for more than two decades, ${ }^{11}$ has recently attracted a great deal of interest. In particular, lead-based iodides $\mathrm{MAPbl}_{3}$ and $\mathrm{FAPbl}_{3}$ $\left(\mathrm{MA}=\mathrm{CH}_{3} \mathrm{NH}_{3}^{+}\right.$and $\mathrm{FA}=\mathrm{CH}\left(\mathrm{NH}_{2}\right)_{2}^{+}$are methylammonium and formamidinium ions) show outstanding performance in solar cell applications and may be synthesised by straightforward processing methods such as spin-coating, dip-coating and vapour deposition techniques. ${ }^{12-14}$ The electrical power conversion efficiency of perovskite devices has shot up markedly and now reached $21.1 \%{ }^{15}$ which is believed to be the result of a unique combination of the orbital character of the conduction and valence band (VB) extrema, large absorption coefficient and long carrier diffusion lengths that result in low recombination rates. ${ }^{16,17}$
It was also suggested that material stability and defect tolerance in these perovskites as well as in other organic-inorganic frameworks may be related to the presence of van der Waals interactions and hydrogen bonding. ${ }^{18-20}$ Recent quantum molecular dynamics simulations indicated that iodine atoms form hydrogen bonds with $\mathrm{H}$ atoms located at the nearest $\mathrm{MA}^{+}$ion, i.e., charge fluctuations due to continuous formation and breakage of these bonds significantly contribute to the dielectric function of $\mathrm{MAPbl}_{3}{ }^{21}$

Motivated by these latest discoveries, we predict the possibility of tuning electronic properties of hybrid organic-inorganic materials and stabilising them by hydrogen bonds, or electrostatic binding of hydrogen to a nearby highly electronegative atom $(\mathrm{N}, \mathrm{O}, \mathrm{F}$, etc). This type of bonding occurs in both inorganic (water) and organic (DNA and proteins) molecules and has an important role in determining the three-dimensional structures adopted by these molecules such as double-helical DNA structure. Many polymers (e.g., nylon) are also strengthened by hydrogen bonds in their main chains. Here we are considering several groups of hybrid organic-inorganic materials in which hydrogen bonds have a significant role in: (i) tuning the band structure of the material (e.g., the orbital character of electronic bands near the bandgap) by forming new bonds and changing hybridisation between the orbitals, and; (ii) stabilising the framework structure to be more resistant to structural transformations. The considered organic-inorganic materials are: (i) the three-dimensional (3D) tetragonal perovskite structures ( $\beta$ - phase) of $\mathrm{H}_{3} \mathrm{SPbX}_{3}$ $\left(\mathrm{X}=\mathrm{I}^{-}, \mathrm{Br}^{-}, \mathrm{Cl}^{-}, \mathrm{F}^{-}\right)$containing the sulfonium cation $\mathrm{H}_{3} \mathrm{~S}^{+}$; (ii) The structural $\delta$-phase of the same materials containing a planar two-dimensional (2D) structure formed by $\mathrm{PbX}_{6}$ octahedra with common edges in their equatorial planes, and; (iii) The 2D material with experimentally known $\mathrm{RbPbF}_{3}$ structure in which $\mathrm{Rb}^{+}$cation is substituted by a protonated sulfonium $\mathrm{H}_{3} \mathrm{~S}^{+}$cation that forms hydrogen bonds with fluorine.

${ }^{1}$ Qatar Environment \& Energy Research Institute (QEERI), Hamad Bin Khalifa University, Doha, Qatar; ${ }^{2}$ College of Science and Engineering, Hamad Bin Khalifa University, Doha, Qatar and ${ }^{3}$ Department of Chemistry and Physics, Birck Nanotechnology Center, Purdue University, West Lafayette, IN, USA.

Correspondence: SN Rashkeev (srashkeev@qf.org.qa)

Received 9 June 2016; revised 20 September 2016; accepted 28 September 2016 


\section{RESULTS}

We performed first-principles density functional theory-based calculations (see details in the Materials and methods section) for crystalline unit cells of the hybrid 2D and 3D organic-inorganic materials mentioned in the previous paragraph and studied the character of wave functions of the states near the bandgap. For this purpose, we projected the density of states (DOSs) onto atomic orbitals of different angular momenta $(s, p$ and $d)$ centred at different atoms. Figure 1 shows the tetragonal perovskite structure ( $\beta$-phase) of $\mathrm{H}_{3} \mathrm{SPbl}_{3}$ (Figure 1a), the band structure of $\mathrm{H}_{3} \mathrm{SPbCl}_{3}$ (Figure $1 \mathrm{~b}$ ), and DOSs of four perovskite materials $\mathrm{H}_{3} \mathrm{SPbX}_{3}\left(\mathrm{X}=\mathrm{I}^{-}, \mathrm{Br}^{-}, \mathrm{Cl}^{-}, \mathrm{F}^{-}\right)$projected onto different atomic species (Figure $1 \mathrm{C}-\mathrm{f}$ ). Analysis of the projected DOS in the vicinity of the bandgap indicates that sulfonium cation makes a significant difference in the orbital character of states near the bandgap as compared with $\mathrm{MAPbl}_{3}$ and $\mathrm{FAPbl}_{3}$ (Figure 2a-d). In $\mathrm{MAPbl}_{3}$, the electronic bandgap is formed between the antibonding top of the $\mathrm{VB}$ originating from the $\mathrm{Pb}(6 s)-\mathrm{I}(5 p)$ interactions and the antibonding conduction band minimum resulting from the $\mathrm{Pb}$ $(6 p)-I(5 p)$ interactions. The $\mathrm{MA}^{+}$cation does not introduce any states at the band edges (the partial contributions from $\mathrm{C}, \mathrm{N}$ and $\mathrm{H}$ atomic orbitals at the energy interval of $5 \mathrm{eV}$ below the VB maximum do not exceed $10^{-3}$ of the total DOS, see ref. 22 and Figure 2e), which could be the reason of high tolerance of this material to some defects. ${ }^{17}$

In the $\mathrm{H}_{3} \mathrm{SPbX}_{3}$ perovskites, the value of the bandgap (Table 1) is mainly defined by the size of the anion (the smallest for $\mathrm{F}^{-}$and largest for $\mathrm{I}^{-}$) and its electronegativity (the lowest for $\mathrm{I}^{-}$and highest for $\left.\mathrm{F}^{-}\right)_{1}{ }^{23}$ which is similar to the $\mathrm{MAPbX}_{3}$ materials. ${ }^{24-28}$ However, in the $\mathrm{H}_{3} \mathrm{SPbX}_{3}$ perovskites, one could observe much higher contributions of the sulfur $(\mathrm{S})$ and hydrogen $(\mathrm{H})$ states in the energy range of $0.5-4 \mathrm{eV}$ below the top of the VB, which means that sulfonium cation orbitals are hybridising with the halogen anion $\mathrm{X}(p)$ orbitals that provide the major contribution to the DOS in this area. This hybridisation is higher for anions with higher electronegativity (or smaller anion radius) being the largest a

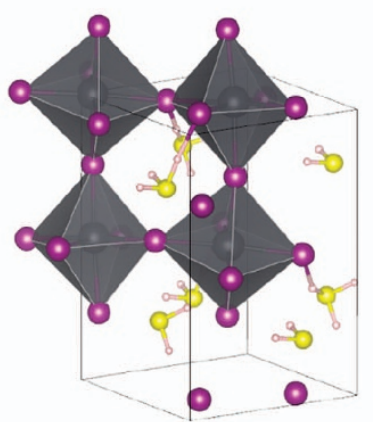

c

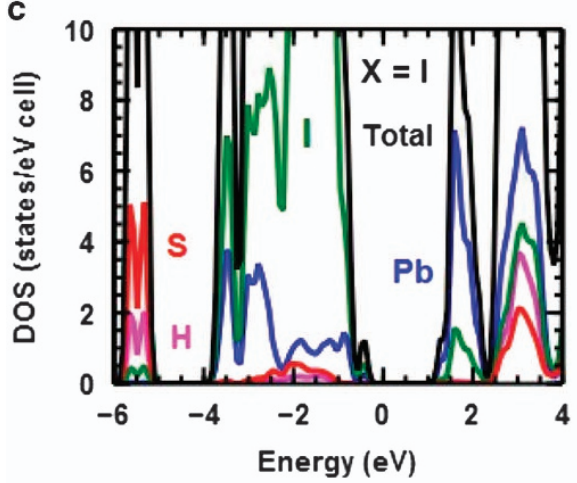

e

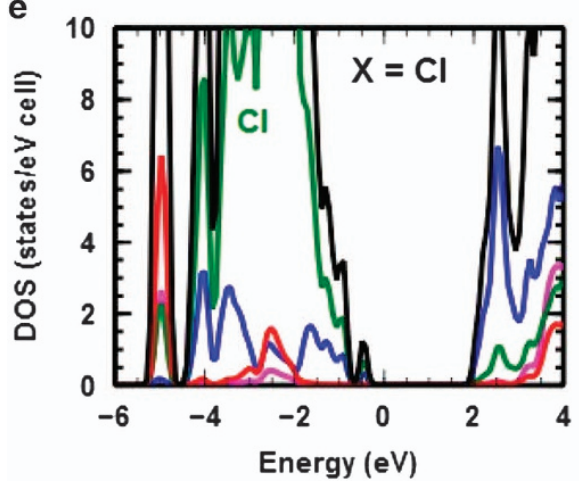

b

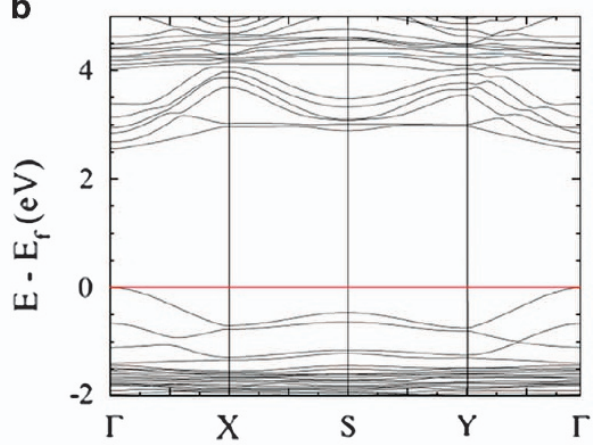

d
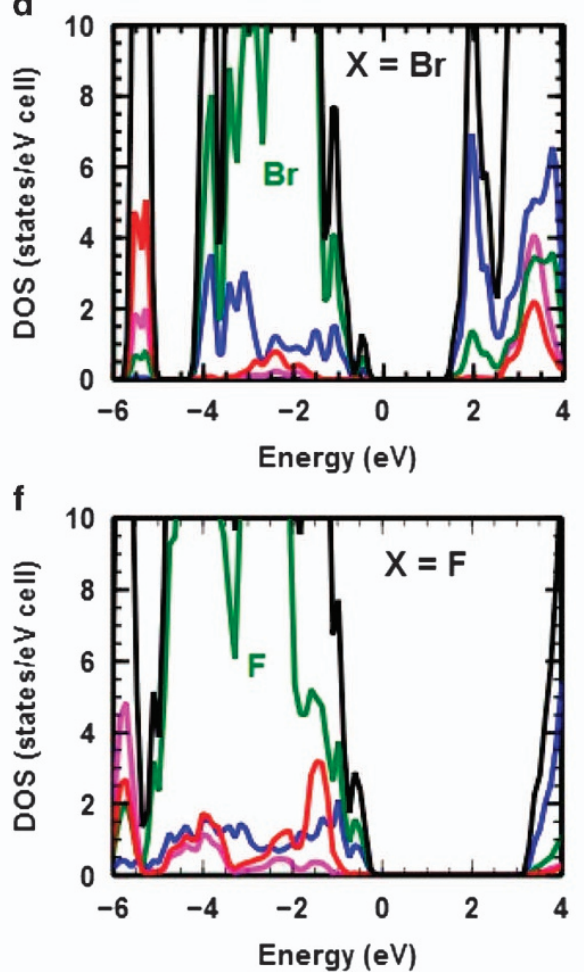

Figure 1. Crystal structure and electronic properties of perovskite $\mathrm{H}_{3} \mathrm{SPbX}_{3}$ materials. (a) The schematic of periodic unit cell of $\mathrm{H}_{3} \mathrm{SPbl}{ }_{3}$ tetragonal perovskite ( $\beta$-phase). $\mathrm{Pb}$ atoms are shown in grey, $\mathrm{I}$-in purple, $\mathrm{S}$-in yellow, $\mathrm{H}$-in white. (b) Electronic band structure of tetragonal $\mathrm{H}_{3} \mathrm{SPbCl}_{3}$. Zero of energy is positioned at the top of the valence band. (c-f) Density of states (total and projected at different atomic species) for $\beta$-phase of $\mathrm{H}_{3} \mathrm{SPbl}_{3}, \mathrm{H}_{3} \mathrm{SPbBr}_{3}, \mathrm{H}_{3} \mathrm{SPbCl}_{3}$ and $\mathrm{H}_{3} \mathrm{SPbF}_{3}$, respectively. Black line indicates the total DOS; green, blue, red and magenta - partial contributions of halogen $(\mathrm{X})$, lead $(\mathrm{Pb})$, sulfur $(\mathrm{S})$ and hydrogen $(\mathrm{H})$, respectively. 
a

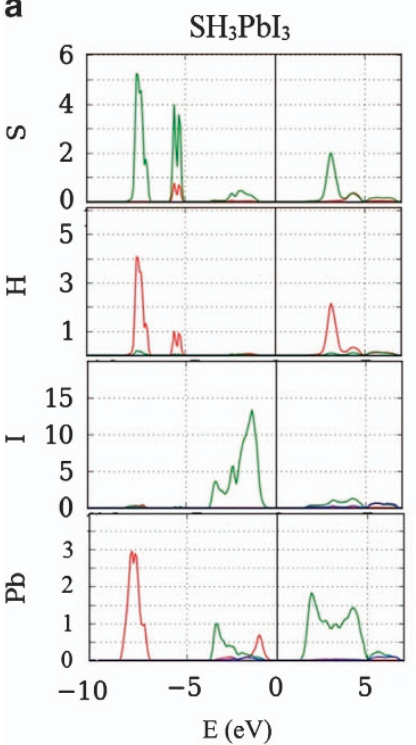

d

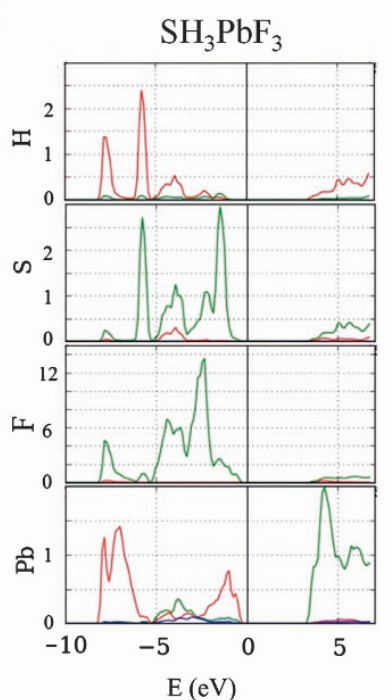

b

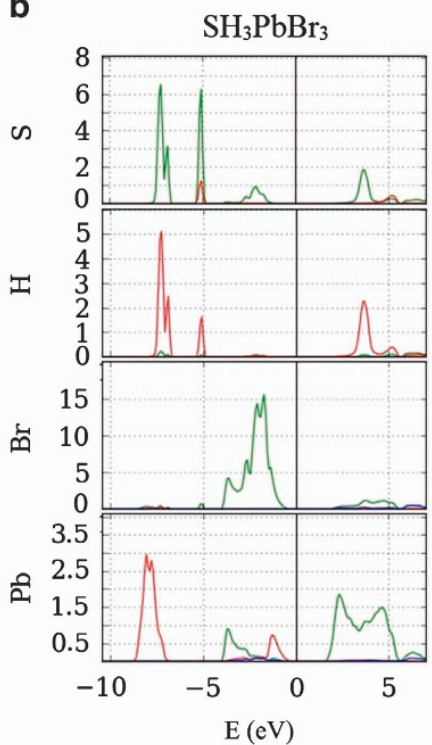

C

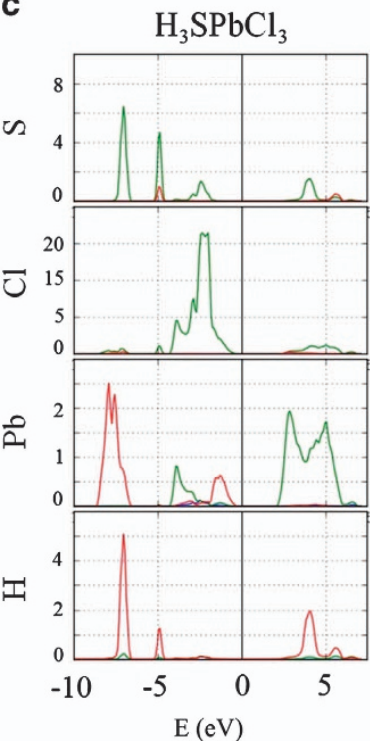

e

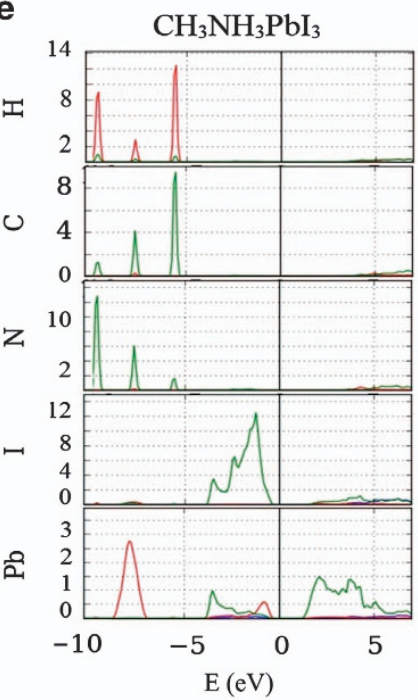

Figure 2. (a-d) DOS for $\mathrm{H}_{3} \mathrm{SPbX}_{3}(\mathrm{X}=\mathrm{I}, \mathrm{Br}, \mathrm{Cl}$ and $\mathrm{F}$ ) materials in $\beta$-phase projected at different atomic species and different angular momentum ( $s, p$ and $d$ ) orbitals. (e) PDOS for $\mathrm{MAPbl}_{3}$ (shown for comparison). Red, green and blue lines indicate the contributions of $s, p$ and $d$ orbitals, respectively.

Table 1. Calculated electronic bandgaps for $\mathrm{H}_{3} \mathrm{SPbX}_{3}(\mathrm{X}=\mathrm{I}, \mathrm{Br}, \mathrm{Cl}$ and F) materials in $\beta$ - and $\delta$-phases

\begin{tabular}{lccccc}
\hline Halogen atom $(X)$ & $I$ & $\mathrm{Br}$ & $\mathrm{Cl}$ & $\mathrm{F}$ \\
\hline Bandgap (eV) in $\beta$-phase (3D) & 1.73 & 2.06 & 2.50 & 3.78 \\
Bandgap (eV) in $\delta$-phase (2D) & 2.49 & 2.88 & 3.44 & 3.76 \\
\hline Abbreviations: 2D, two dimensional; 3D, three dimensional. \\
\hline
\end{tabular}

for $\mathrm{H}_{3} \mathrm{SPbF}_{3}$ and lowest for $\mathrm{H}_{3} \mathrm{SPbl}_{3}$ (Figure 1c-f). The hybridised $\mathrm{H}_{3} \mathrm{~S}-\mathrm{X}$ states form additional features at the DOS curves-one or more noticeable shoulders or even local peaks near the top of the VB. An analysis based on the projected DOS onto atomic orbitals of different angular momenta centred at different atoms (Figure 2) shows that these features are related to the hybridisation of $\mathrm{S}(3 p)-\mathrm{H}(1 s)-\mathrm{X}(p)$ orbitals, which increases energy distances between the upper VBs (Figure $1 \mathrm{~b}$ ).
In the structural $\delta$-phases of $\mathrm{H}_{3} \mathrm{SPbX}_{3}$ materials that crystallise in unit cell four times larger than the perovskite unit cell, the planar $2 \mathrm{D}$ structure is formed by $\mathrm{PbX}_{6}$ octahedra that share edges in their equatorial planes (Supplementary Figure S1a). We found that the qualitative behaviour of $\delta$-modifications of these materials is very similar to the behaviour of their 3D perovskite counterparts, i.e., the $\delta$-phase DOSs projected onto different atomic species also indicate a significant admixture of the $\mathrm{S}$ and $\mathrm{H}$ orbitals of the sulfonium ions near the top of the VB (Supplementary Figure S1). The bandgaps for the 2D $\delta$-phase materials containing the $\mathrm{I}^{-}, \mathrm{Br}^{-}$and $\mathrm{Cl}^{-}$ions are higher than in same materials in the $3 \mathrm{D}$ $\beta$-phase, whereas for $\mathrm{H}_{3} \mathrm{SPbF}_{3}$, the bandgaps are similar for both 2D and 3D phases (Table 1).

We also performed calculations for the $2 \mathrm{D}$ material $\mathrm{H}_{3} \mathrm{SPbF}_{3}$ that crystallises in experimentally known $\mathrm{RbPbF}_{3}$ structure, in which $\mathrm{Rb}^{+}$cations are substituted by protonated $\mathrm{H}_{3} \mathrm{~S}^{+}$cations. The structure of this material is different from the $\delta$-phase structures considered above-here the $2 \mathrm{D}$ planes consist of $\mathrm{PbF}_{4}$ tetrahedra (instead of octahedra in the $\delta$-phase) that have common edges (Figure 3a). We found that $\mathrm{H}_{3} \mathrm{~S}$ molecules in this framework 
also form hydrogen bonds with fluorine anions, and the electronic structure near the top of VB is similar to the two other groups of materials considered earlier-in addition to the fluorine $p$-states, there is a strong admixture of the $\mathrm{S}(3 p)$ and $\mathrm{H}(1 s)$ states that form two extra peaks in the DOS near the top of VB (Figure $3 b$ ). Both of these peaks are related to the formation of $\mathrm{S}-\mathrm{H}-\mathrm{F}$ bridges and are not observed in the original material with more isotropic $\mathrm{Rb}^{+}$cations. Figure $3 \mathrm{c}$ shows that these peaks should be observed in the imaginary part of the dielectric function, $\varepsilon_{2}(\omega)$, at ultraviolet energies $\sim 4.5$ and $5.3 \mathrm{eV}$ for any possible polarisation of the electric field (i.e., they will be also observed in polycrystalline samples and/or in unpolarised light experiments).
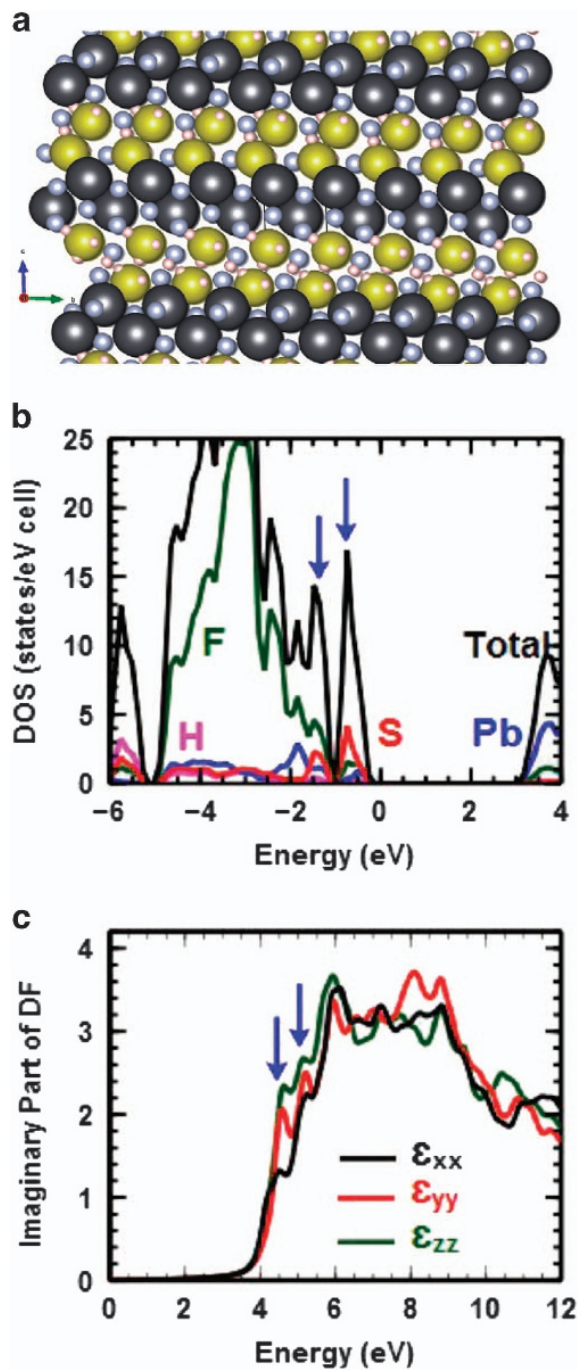

Figure 3. Electronic properties of $\mathrm{H}_{3} \mathrm{SPbF}_{3}$ material crystallising in the $\mathrm{RbPbF}_{3}$ structure. (a) The schematic of $\mathrm{H}_{3} \mathrm{SPbF}_{3}$ crystal structure shown in the space filling mode. $\mathrm{Pb}$ atoms are shown in dark grey, $\mathrm{F}$-in light grey, $\mathrm{S}$-in yellow, $\mathrm{H}$-in white. (b) Electronic density of states (total and projected at different atomic species) of $\mathrm{H}_{3} \mathrm{SPbF}_{3}$, material crystallising in $\mathrm{RbPbF}_{3}$ structure. Black line indicates the total DOS; green, blue, red and magenta-partial contributions of fluorine $(\mathrm{F})$, lead $(\mathrm{Pb})$, sulfur $(\mathrm{S})$ and hydrogen $(\mathrm{H})$. (c) Calculated imaginary part of the dielectric function (DF) for this material for three different polarisations of the electric field. Vertical blue arrows indicate interband electronic transitions from electronic states positioned near the top of VB (also indicated with blue arrows in b) to the edge of the conduction band.

\section{DISCUSSION}

All these results support the assumption that protonated sulfonium cation $\mathrm{H}_{3} \mathrm{~S}^{+}$interacts with halogen anion, the interaction being stronger for anions with higher electronegativity $\left(\mathrm{Cl}^{-}\right.$and $\left.\mathrm{F}^{-}\right)$. To understand the nature of this interaction, we plotted the electron localisation function contours ${ }^{29}$ for several considered materials in the vicinity of the $\mathrm{H}_{3} \mathrm{~S}-\mathrm{X}$ complexes. In perovskite structure, the electron localisation function minimum between the $\mathrm{H}_{3} \mathrm{~S}$ complex and the iodine atom corresponds to the absence of bonding between them while an $\mathrm{S}-\mathrm{H}-\mathrm{F}$ electronic bridge is formed with fluorine (Figure $4 a, b$ ). This is a typical example of hydrogen bonding in which hydrogen atom electrostatically attracts to strongly electronegative fluorine. This hydrogen bond has some features of a covalent bond as well-it is directional and forms interatomic distances shorter than the sum of the van der Waals radii. An even stronger $\mathrm{H}-\mathrm{F}$ hydrogen bond is observed in the $\mathrm{H}_{3} \mathrm{SPbF}_{3}$ material with $\mathrm{RbPbF}_{3}$ structure (Figure 4c), which means that $\mathrm{S}-\mathrm{H}-\mathrm{F}$ bridges could be observed in

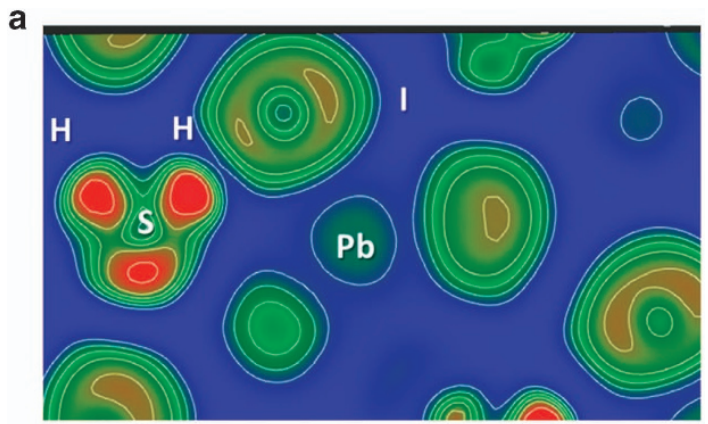

b
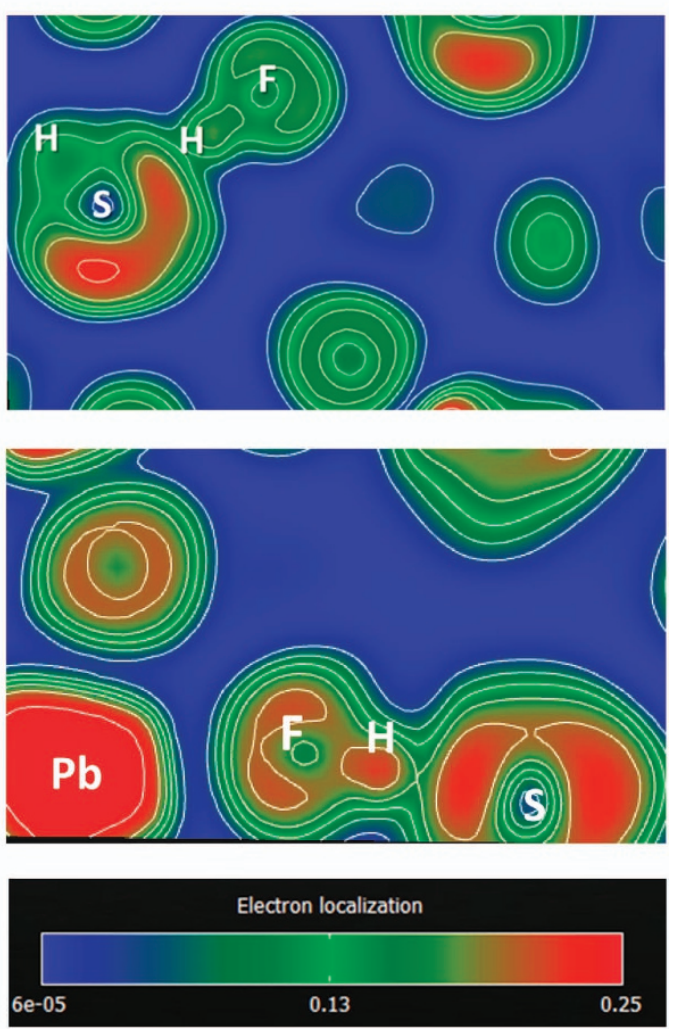

Figure 4. Electron localization function (ELF) contours of different $\mathrm{H}_{3} \mathrm{SPbX}_{3}$ materials in the vicinity of $\mathrm{H}_{3} \mathrm{~S}-\mathrm{X}$ complexes. (a) ELF for $\mathrm{H}_{3} \mathrm{SPbl}_{3}$ in perovskite structure ( $\beta$-phase). (b) ELF for $\mathrm{H}_{3} \mathrm{SPbF}_{3}$ in perovskite structure. (c) ELF for $\mathrm{H}_{3} \mathrm{SPbF}_{3}$ crystallising in $\mathrm{RbPbF}_{3}$ structure. 
Table 2. Calculated reaction, formation and Hull energies for $\mathrm{H}_{3} \mathrm{SPbX}_{3}$ $(\mathrm{X}=\mathrm{I}, \mathrm{Br}, \mathrm{Cl}$, and $\mathrm{F}$ ) materials in $\beta$ - and $\delta$-phases (in eV per cell)

\begin{tabular}{|c|c|c|c|}
\hline Material & 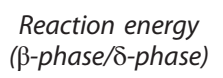 & 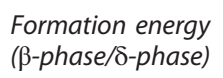 & 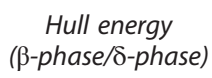 \\
\hline $\mathrm{H}_{3} \mathrm{SPbl}_{3}$ & $-0.21 /-0.18$ & $-0.48 /-0.44$ & $-0.30 /-0.26$ \\
\hline $\mathrm{H}_{3} \mathrm{SPbBr}_{3}$ & $-0.16 /-0.14$ & $-0.63 /-0.61$ & $-0.25 /-0.23$ \\
\hline $\mathrm{H}_{3} \mathrm{SPbCl}_{3}$ & $-0.10 /-0.08$ & $-0.97 /-0.96$ & $-0.18 /-0.17$ \\
\hline $\mathrm{H}_{3} \mathrm{SPbF}_{3}$ & $0.01 /-0.02$ & $-1.55 /-1.58$ & $-0.07 /-0.28$ \\
\hline
\end{tabular}

Reaction energies were calculated for the reactions: $\mathrm{PbS}+3 \mathrm{HX} \rightarrow \mathrm{H}_{3} \mathrm{SPbX}_{3}$.

many inorganic-organic frameworks containing sulfonium cations and highly electronegative halide anions.

The high $\mathrm{S}-\mathrm{H}-\mathrm{F}$ angle of $167-177^{\circ}$ observed in all considered materials indicates that there is competition between the $\mathrm{S}-\mathrm{H}$ covalent bond and hydrogen attraction to electronegative anion (Cl or F). Most likely, such a behaviour has even more universal character and could be observed in materials containing protonated cations based on atoms with electronegativity lower than nitrogen (e.g., phosphorus). The length of the $\mathrm{N}-\mathrm{H}$ covalent bond $(1.04 \AA)$ is much lower than of the $\mathrm{S}-\mathrm{H}$ and $\mathrm{P}-\mathrm{H}$ bonds $(1.34$ and $1.44 \AA$ ), which is an important geometric factor explaining why protonated amines (e.g., MA and FA, for which most of the previous studies on inorganic-organic photovoltaic devices have been performed) prefer not to form hydrogen bonds with surrounding atoms even with those with high electronegativity. In sulfates and phosphates, however, this restriction is lifted, which provides an opportunity to form extra hydrogen bonds that could: (i) contribute in forming the unique structures of the network; (ii) increase their stability; and (iii) provide fine tuning of the states at the edges on the bandgap.

We also found that most of the considered hybrid inorganicorganic materials are stable. In addition to the formation energy, we calculated the reaction energy (the difference between the total energy of reaction products and reactants) $)^{30}$ and the Hull energy (the difference in formation energies which evaluates the stability of a given compound against any linear combination of compounds that have the same averaged composition) $)^{31-33}$ for $\beta$ - and $\delta$-phases (Table 2). The results for $\mathrm{H}_{3} \mathrm{SPbX}_{3}(X=\mathrm{I}, \mathrm{Br}, \mathrm{Cl}$ and $F)$ materials show that all of these energies are negative except the reaction energy for the $\beta$-phase of $\mathrm{H}_{3} \mathrm{SPbF}_{3}$ that is zero within the accuracy of the calculations.

\section{SUMMARY}

In conclusion, we demonstrate that tuning electronic properties of hybrid organic-inorganic complex materials may be achieved using protonated cations that form hydrogen bonds between the cation and surrounding octahedra. In particular, the value of the bandgap in the $\mathrm{H}_{3} \mathrm{SPbX}_{3}(\mathrm{X}=\mathrm{I}, \mathrm{Br}, \mathrm{Cl}$ and $\mathrm{F})$ materials is defined by the size of the anion and the electronegativity of materials constituents that results in electrostatic interactions. Hydrogen bonding also makes these materials stable, which is promising for many applications. We suggest that such a behaviour should be observed in different hybrid inorganic-organic frameworks containing protonated cations.

The potential tunability of the states near the edges of the bandgap (VB maximum and conduction band minimum) could also provide a unique tool for developing different photovoltaic and optoelectronic devices including solar cells, light emission diodes, electrically driven lasers, nonlinear optical materials and systems, and charge storages (see review articles ${ }^{24-26,28,34}$ for more details). Hydrogen bonding mixes up the states of the protonated cation and the anion that gives a contribution to DOS and optical absorption spectra at specific frequencies close to the bandgap threshold (peaks or shoulders) that could be identified and selectively excited by laser. This could offer a new approach to different devices with promising applications in nanoelectronics, optics and data storages.

\section{MATERIALS AND METHODS}

We employ first-principles calculations to evaluate the electronic structure and estimate the stability of the proposed materials. Density functional theory calculations are performed with the projector augmented wave method as implemented in the Vienna Ab-initio Simulation Package. ${ }^{35}$ All calculations were performed using spin-polarised generalised gradient approximation with the Perdew-Burke-Ernzerhof parameterisation for the exchange and correlation energy of interacting electrons. ${ }^{36}$ The energy cutoff for the planewave basis set was set to $520 \mathrm{eV}$ and a $8 \times 8 \times 8$ Monkhorst-Pack k-point mesh was employed. Long-range van der Waals interactions have been taken into account via the Tkatchenko and Scheffler scheme. ${ }^{37}$ The convergence of the final forces is set to $0.01 \mathrm{eV} / \mathrm{A}$.

\section{ACKNOWLEDGEMENTS}

The simulations were performed at the Research Computing Center in Texas A\&M University at Qatar and the Swiss Supercomputing Center (CSCS).

\section{CONTRIBUTIONS}

All authors designed the research. FEM performed calculations. All participated in data analysis and writing the paper.

\section{COMPETING INTERESTS}

The authors declare no conflict of interest.

\section{REFERENCES}

1. James, S. L. Metal-organic frameworks. Chem. Soc. Rev. 32, 276-288 (2003).

2. Bertani, R. et al. Halogen bonding in metal-organic-supramolecular networks. Coordination Chemistry Reviews 254, 677-695 (2010).

3. Li, B., Zang, S.-Q., Wang, L. Y. \& Mak, T. C. W. Halogen bonding: a powerful emerging tool for constructing high-dimensional metal-containing supramolecular networks. Coord. Chem. Rev. 308, 1-21 (2016).

4. Leblanc, N., Allain, M., Mercier, N. \& Cariati, E. Protonated N,N'-Dioxide4,4'-bipyridine, an interesting synthon for the building of polar $\mathrm{H}$-bonded networks? Cryst. Growth Des. 11, 5200-5205 (2011).

5. Kreno, L. E. et al. Metal-organic framework materials as chemical sensors. Chem. Rev. 112, 1105-1125 (2012).

6. Morris, R. E. \& Wheatley, P. S. Gas storage in nanoporous materials. Angew. Chem. Intern. Ed. 47, 4966-4981 (2008).

7. Czaja, A. U., Trukhan, N. \& Muller, U. Industrial applications of metal-organic frameworks. Chem. Soc. Rev. 38, 1284-1293 (2009).

8. Cheetham, A. K. \& Rao, C. N. R. There's room in the middle. Science 318, 58-59 (2007).

9. Di Sante, D., Stroppa, A., Jain, P. \& Picozzi, S. Tuning the Ferroelectric Polarization in a Multiferroic Metal-Organic Framework. J. Am. Chem. Soc. 135, 18126-18130 (2013).

10. Weng, D.-F., Wang, Z.-M. \& Gao, S. Framework-structured weak ferromagnets. Chem. Soc. Rev. 40, 3157-3181 (2011).

11. Mitzi, D. B., Feild, C. A., Harrison, W. T. A. \& Guloy, A. M. Conducting tin halides with a layered organic-based perovskite structure. Nature 369 467-469 (1994).

12. Burschka, J. et al. Sequential deposition as a route to high-performance perovskite-sensitized solar cells. Nature 499, 316-319 (2013).

13. Liu, M., Johnston, M. B. \& Snaith, H. J. Efficient planar heterojunction perovskite solar cells by vapour deposition. Nature 501, 395-398 (2013).

14. Eperon, G. E. et al. Formamidinium lead trihalide: a broadly tunable perovskite for efficient planar heterojunction solar cells. Energy Environ. Sci. 7, 982-988 (2015).

15. Saliba, M. et al. Cesium-containing triple cation perovskite solar cells: improved stability, reproducibility and high efficiency. Energy Environ. Sci. 9, 1989-1997 (2016). 
16. Kim, H. S., Im, S. H. \& Park, N. G. Organolead halide perovskite: new horizons in solar cell research. J. Phys. Chem. C 118, 5615-5625 (2014).

17. Brandt, R. E., Stevanovic, V., Ginley, D. S. \& Buonassisi, T. Identifying defect-tolerant semiconductors with high minority-carrier lifetimes: beyond hybrid lead halide perovskites. MRS Commun. 5, 265-275 (2015).

18. Berry, J. et al. Hybrid organic-inorganic perovskites (HOIPs): opportunities and challenges. Adv. Mater. 27, 5102-5112 (2015).

19. Mitzi D. in Progress in Inorganic Chemistry (ed. Karlin, K. D.) Vol. 48 1-121 (John Wiley \& Sons, Inc., 1999).

20. Tan, J. C. \& Cheetham, A. K. Mechanical properties of hybrid inorganic-organic framework materials: establishing fundamental structure-property relationships. Chem. Soc. Rev. 40, 1059-1080 (2011).

21. Hakamata, T. et al. The nature of free-carrier transport in organometal halide perovskites. Sci. Rep. 6, 19599 (2016).

22. Wang, Y. et al. Density functional theory analysis of structural and electronic properties of orthorhombic perovskite $\mathrm{CH}_{3} \mathrm{NH}_{3} \mathrm{Pbl}_{3}$. Phys. Chem. Chem. Phys. 16, 1424-1429 (2014).

23. Castelli, I. E., García-Lastra, J. M., Thygesen, K. S. \& Jacobsen, K. W. Bandgap calculations and trends of organometal halide perovskites. APL Mater. 2, 081514 (2014)

24. Stranks, S. D. \& Snaith, H. J. Metal-halide perovskites for photovoltaic andlight-emitting devices. Nat. Nanotechnol. 10, 391-402 (2015).

25. Saparov, B. \& Mitzi, D. B. Organic-inorganic perovskites: structural versatility for functional materials design. Chem. Rev. 116, 4558-4596 (2016).

26. Mitzi, D. B., Chondroudis, K. \& Kagan, C. R. Organic-inorganic electronics. IBM J. Res. Dev. 45, 29-45 (2001)

27. Berdiyorov, G. R. et al. Effect of halide-mixing on the electronic transport properties of organometallic perovskites. Sol. Energy Mater. Sol. Cells 148, 2-10 (2016)

28. Manser, J. S., Christians, J. A. \& Kamat, P. V. Intriguing optoelectronic properties of metal halide perovskites. Chem. (e-pub ahead of print 21 June 2016; doi:10.1021/acs.chemrev.6b00136).

29. Becke, A. D. \& Edgecombe, K. E. A simple measure of electron localization in atomic and molecular systems. J. Phys. Chem. 92, 5397-5403 (1990).
30. Hautier, G., Ong, S. P., Jain, A., Moore, C. J. \& Ceder, G. Accuracy of density functional theory in predicting formation energies of ternary oxides from binary oxides and its implication on phase stability. Phy. Rev. B 85, 155208 (2012).

31. Castelli, I. E., Garcia-Lastra, J. M., Huser, F., Thygesen, K. S. \& Jacobsen, K. W. Stability and bandgaps of layered perovskites for one-and two-photon water splitting. New J. Phy. 15, 105026 (2013).

32. d'Avezac, M. \& Zunger, A. Identifying the minimum-energy atomic configuration on a lattice: Lamarckian twist on darwinian evolution. Phy. Rev. B 78, 064102 (2008).

33. Trimarchi, G., Freeman, A. J. \& Zunger, A. Predicting stable stoichiometries of compounds via evolutionary global space-group optimization. Phy. Rev. B 80, 092101 (2009)

34. Lee, J.-W., Kim, H.-S. \& Park, N.-G. in Organic-Inorganic Halide Perovskite Photovoltaics (eds Park, N.-G. et al.) 223-253 (Springer International Publishing, 2016).

35. Kresse, G. \& Furthmuller, J. Efficient Iterative Schemes for Ab-initio Total-Energy Calculations Using a Plane-Wave Basis Set. Phys. Rev. B 54, 11169-11186 (1996).

36. Perdew, J. P., Burke, K. \& Ernzerhof, M. Generalized gradient approximation made simple. Phys. Rev. Lett. 77, 3865-3868 (1996).

37. Tkatchenko, A., Di Stasio, R. A., Car, R. \& Scheffler, M. Accurate and efficient method for many-body van der Waals interactions. Phys. Rev. Lett. 108, 236402 (2012).

\section{cc) (i)}

This work is licensed under a Creative Commons Attribution 4.0 International License. The images or other third party material in this article are included in the article's Creative Commons license, unless indicated otherwise in the credit line; if the material is not included under the Creative Commons license, users will need to obtain permission from the license holder to reproduce the material. To view a copy of this license, visit http://creativecommons.org/licenses/ by/4.0/

(c) The Author(s) 2016

Supplementary Information accompanies the paper on the npj Computational Materials website (http://www.nature.com/npjcompumats) 